\title{
Evaluation of microfluidic channels with optical coherence tomography
}

\author{
J. Czajkowski ${ }^{a}$, T. Prykäri ${ }^{a}$, E. Alarousu $^{b}$, J. Lauri $^{a}$, and R. Myllylä ${ }^{a}$ \\ ${ }^{a}$ Optoelectronics and Measurement Techniques Laboratory, University of Oulu, P.O. Box 4500, \\ 90014 University of Oulu, Finland \\ ${ }^{b}$ Solar and Photovoltaics Engineering Research Center, King Abdullah University of Science \\ and Technology, P.O.Box 55455 , Jeddah 21534, Saudi Arabia
}

\begin{abstract}
Application of time domain, ultra high resolution optical coherence tomography (UHR-OCT) in evaluation of microfluidic channels is demonstrated. Presented study was done using experimental UHR-OCT device based on a Kerr-lens mode locked Ti:sapphire femtosecond laser, a photonic crystal fibre and modified, free-space Michelson interferometer. To show potential of the technique, microfluidic chip fabricated by VTT Center for Printed Intelligence (Oulu, Finland) was measured. Ability for full volumetric reconstruction in non-contact manner enabled complete characterization of closed entity of a microfluidic channel without contamination and harm for the sample. Measurement, occurring problems, and methods of postprocessing for raw data are described. Results present completely resolved physical structure of the channel, its spatial dimensions, draft angles and evaluation of lamination quality.
\end{abstract}

Keywords: optical metrology, low coherence interferometry, imaging, microfluidics, hot-embossing

\section{INTRODUCTION}

Polymer materials are becoming more and more important in today's research and high volume production. They offer a variety of physical and chemical properties desired by different application fields. Classic, widely used microfluidic structures are fabricated using silicon or glass which, in contrast to polymers, require expensive and slow processing. Important factor favouring polymer microfluidics in applications concerning living cells is permeability to gases. ${ }^{1}$ Additional superiority of polymer-based materials comes from their low electrical conductivity and mechanical flexibility. Glass, silicon and steel remain superior in applications requiring chemical, thermal, and mechanical stability.

In this paper we focus on an example of polymer structures in life science i.e., microfluidic channels. Microfluidics combine small size of structures with ability to exploit behaviour of fluids in microchannels. It offers fundamentally new capabilities in the control of concentrations of molecules in space and time. Microfluidic systems are widely used to screen conditions for protein crystallization, in cell biology, in chemical synthesis, and in biosensing. ${ }^{1}$ Due to many proposed applications and high interest of biomedical societies, microfluidics is rapidly developing research and market branch. As any other field, it requires accurate, easy to use and cost-effective evaluation methods.

Fabrication process of microfluidic chips has several stages. In the first step, a stamp responsible for topology of microfluidic chip is produced. Its width, height, and draft angle govern final dimensions of the channels. Second fabrication stage is hot-embossing.

Features are added to substrate materials using pressure and high temperature. These factors have also great influence on spatial dimensions of the channel. After embossing process, cognitive agents (e.g., enzymes, antigens, antibodies, proteins, receptors etc.) may be introduced. It is also possible to introduce them as a fluid into finalized structure. Complete information about agents and specific applications of microfluidics can be found from references. ${ }^{1,2}$

Further author information: (Send correspondence to:)

Jakub Czajkowski: E-mail: jakub.czajkowski@ee.oulu.fi, Telephone: +358466 818 339, Fax: +358 85532774

Laser Applications in Life Sciences, edited by Matti Kinnunen, Risto Myllylä, Proc. of SPIE Vol. 7376, $737617 \cdot$ ○ 2010 SPIE · CCC code: 0277-786X/10/\$18 · doi: 10.1117/12.871445

Proc. of SPIE Vol. $7376737617-1$ 
During the last fabrication stage, lamination process takes part. Both flat bed and roll-to-roll (R2R) fabrication methods can be used. Each of mentioned fabrication steps is equally important and has effect on quality and properties of final product.

Before the lamination process takes part, hot-embossed structure can be evaluated both in mechanical and optical manner. Toh et. al. used stylus profiler, ${ }^{3}$ Chien applied a 3D laser scanning microscope, ${ }^{4}$ while $\mathrm{Mg}$ et. al. presented measurements done using scanning electron microscope (SEM). ${ }^{5}$ Hardt et. al compared in their report results obtained by interferometric optical profiler, atomic force microscope (AFM), and SEM. ${ }^{6}$ Another group from Massachusetts Institute of Technology presented a review on metrology of microfluidic devices, containing list of critical parameters and example applications. ${ }^{7}$ In the review AFM, interferometry and confocal microscopy were also compared. Results of such structural measurements may be correlated with dimensions of the stamp used for hot-embossing, giving information about process accuracy. Some geometry, surface or sub-surface aspects may be critical for specific microfluidic applications. ${ }^{7}$

After lamination, microfluidic channel becomes a closed entity, which complicates its evaluation. There are means to evaluate its flow and bio-chemical properties, but investigation of physical properties, such as spatial dimensions and lamination quality, remains an issue. Previously presented methods are no longer applicable. This is where optical coherence tomography (OCT) could be used.

OCT has been developed for almost 20 years, ${ }^{8}$ but still non-medical use of this technique is rare. Authors of this paper have been investigating OCT and its application in industry for several years, presenting results obtained with self-developed ultra-high resolution time domain OCT setup. ${ }^{9-17}$ Stifter presented review on alternative applications and developments for optical coherence tomography. ${ }^{18}$

When it comes to measurements of microfluidic chips, one of the most important assets of optical coherence tomography is its non-contact measurement principle. As main application field of microfluidic chips is bio and medical science, this modality plays important role. Sample analyzed with OCT will not be damaged or contaminated during testing. Moreover, OCT offers complete three-dimensional volumetric reconstruction. Therefore presented technique could be used in manufacturing process adjustment, quality control, as well as in simulation or theoretical calculations.

Some application possibilities emerge from fabrication technology of polymer based microfluidic chips. R2R concept used in fabrication process of microfluidic chips is widely used in the whole printing industry. Therefore one can observe its rapid development. ${ }^{19}$ In our recent publication we have already presented possibility to use OCT in evaluation of R2R fabricated printed electronics. ${ }^{17}$ In both cases (i.e., in microfluidics and printed electronics), the core principle of fabrication method is the same, thus it could be possible to create unified, on-line inspection device based on optical coherence tomography.

\section{MEASUREMENT SYSTEM}

Our research team has been developing the UHR-OCT system based on the modified free-space Michelson interferometer. ${ }^{10,12,13,16,17}$ A combination of a Kerr-lens mode locked Ti-sapphire femtosecond laser followed by a photonic crystal fibre $(\mathrm{PCF})$ is used as a light source for the interferometer. Measurement process is controlled by specially designed LabVIEW based program. ${ }^{20}$

In developed system, TISSA-50 (CDP Corp.) femtosecond laser is used. It generates $<50 \mathrm{fs}$ pulses with centre wavelength around $800 \mathrm{~nm}$. Light travels through dispersion compensation path consisting of two prisms and two mirrors. Laser beam is then coupled to the photonic crystal fibre (PCF, Crystal Fibre Femtowhite 800) and is broadened in so called supercontinuum generation process. High output power (around $130 \mathrm{~mW}$ after the PCF) and bandwidth range of 400-1700 $\mathrm{nm}$ enables ultra-high resolution OCT imaging. However, because of very broadband light source, exceeding transmission capabilities of today's fibres, free-space Michelson interferometer is used. ${ }^{12,13,17}$

During the measurement an A-scan (i.e. the depth scan) is acquired by movement of piezoelectric scanner in the reference arm. To maintain both interferometer arms in focus during whole duration of an A-scan, second piezoelectric scanner is implemented in the measurement arm, providing dynamic focusing. 


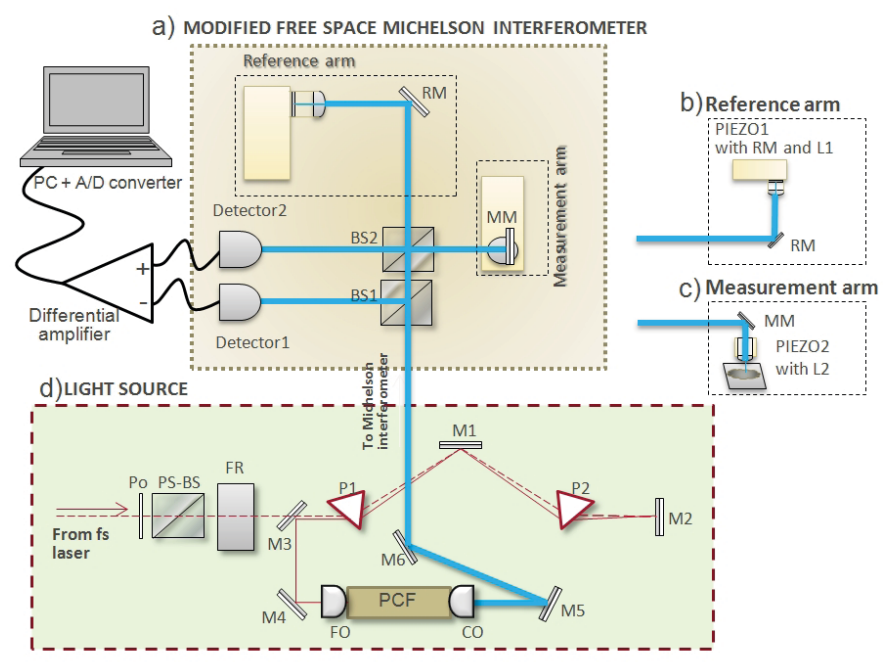

Figure 1. Layout scheme of experimental UHR-OCT device. (a) Michelson type interferometer: BS1, BS2 - beam splitters, RM - reference mirror, MM - measurement mirror, L1-L2 - focusing lenses. (b) reference arm, (c) measurement arm, (d) Light source for the interferometer: Po - polariser, PS-BS - polarization sensitive beam splitter, FR - Faraday's rotator, $\mathrm{P}$ - prisms for dispersion compensation, M - mirror, Fo - focusing objective, Co - collimation objective.

Due to the use of so-called time domain OCT method, balanced detection mode is introduced to assure high SNR ratio. Following the measurement, analogue signal is filtered, digitized and further processed using Matlab and ParaView software. Detailed layout scheme of developed system can be seen in Figure 1. More information about the system and its components can be found in references. ${ }^{10,12,13}$

\section{MEASUREMENT AND DATA POSTPROCESSING}

\subsection{Measurement of microfluidic channel}

For evaluation purpose, area of $1500 \times 1500 \mu \mathrm{m}$ was measured using developed optical coherence tomography system. Each lateral point provided axial vector of 7500 points (A-scan) covering $272 \mu \mathrm{m}$. Sample was measured from substrate's backplane side i.e., opposite to hot-embossing direction. For the measurement region of interest channel's inlet was chosen.

Optical coherence tomography is a refractive index sensitive technique. It means that amplitude of interference fringe signal is proportional to refractive index mismatch. That mismatch provides structural information about the sample. As OCT is based on low coherence interferometry, refractive index has also influence on measured axial distances, thus resolved structure. To provide interference, optical path length difference between photons reflected in sample arm and photons in the reference arm should be less or equal to coherence length of the light source. Let's define refractive index as a factor, by which velocity of light in particular medium is lower than velocity of light in vacuum. Therefore travel time, thus resolved reflection site's depth will be multiplied by this factor. The left part of Figure 2 shows sample tomogram (B-scan image) presenting refractive index problem. Bottom of microfluidic channel's inlet hole seems to be above actual channel. It is then clear that resolved, optical structure does not match physical properties of the sample. The right part of the figure shows regions of interest (ROI) that were used in data processing.

In case of the microfluidic chip, three basic structural areas can be considered. First area is channel's inlet hole, with the bottom of the hole and air above it (i.e., $n=1$ ). Laminated parts of the chip can be considered as another area, where refractive index should be constant (thus no interference is recorded). Finally, the channel itself creates area, where both polymer $(n>1)$ and air $(n=1)$ can be distinguished. All this means that raw measurement data contains distorted optical structure, which has to be corrected to match the physical one. The volumetric reconstruction image of the raw measurement data can be seen in Figure 3. The actual microfluidic channel lays between interfaces of ROI3 and ROI4, whereas ROI2 is the bottom of channel's inlet hole. 

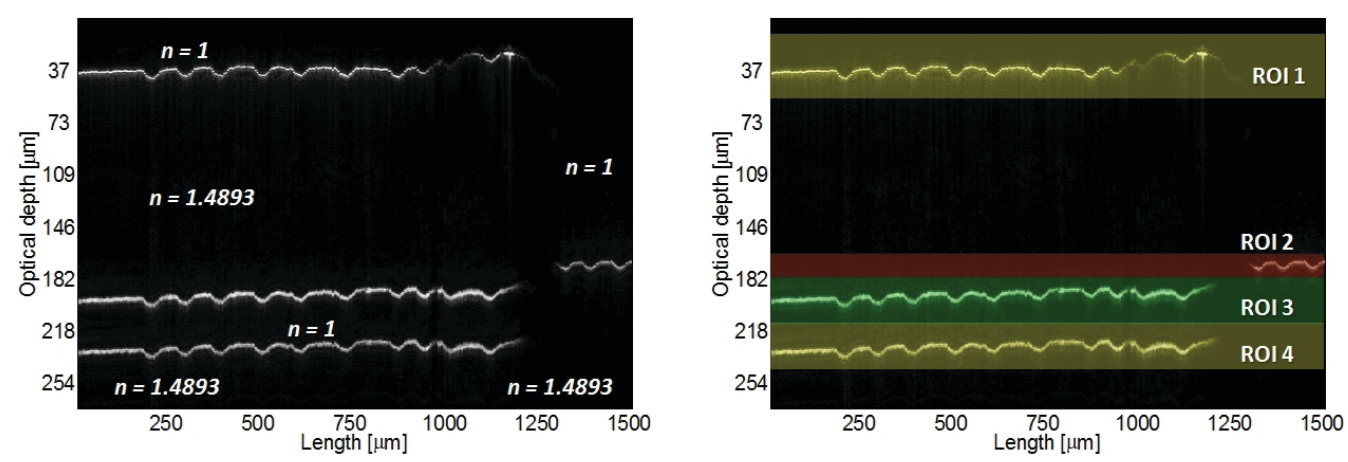

Figure 2. Tomograms (B-scan images) of microfluidic channel. Markings in the left image show refractive indices. Regions of interest for interface detection are marked with rectangles in the right image.

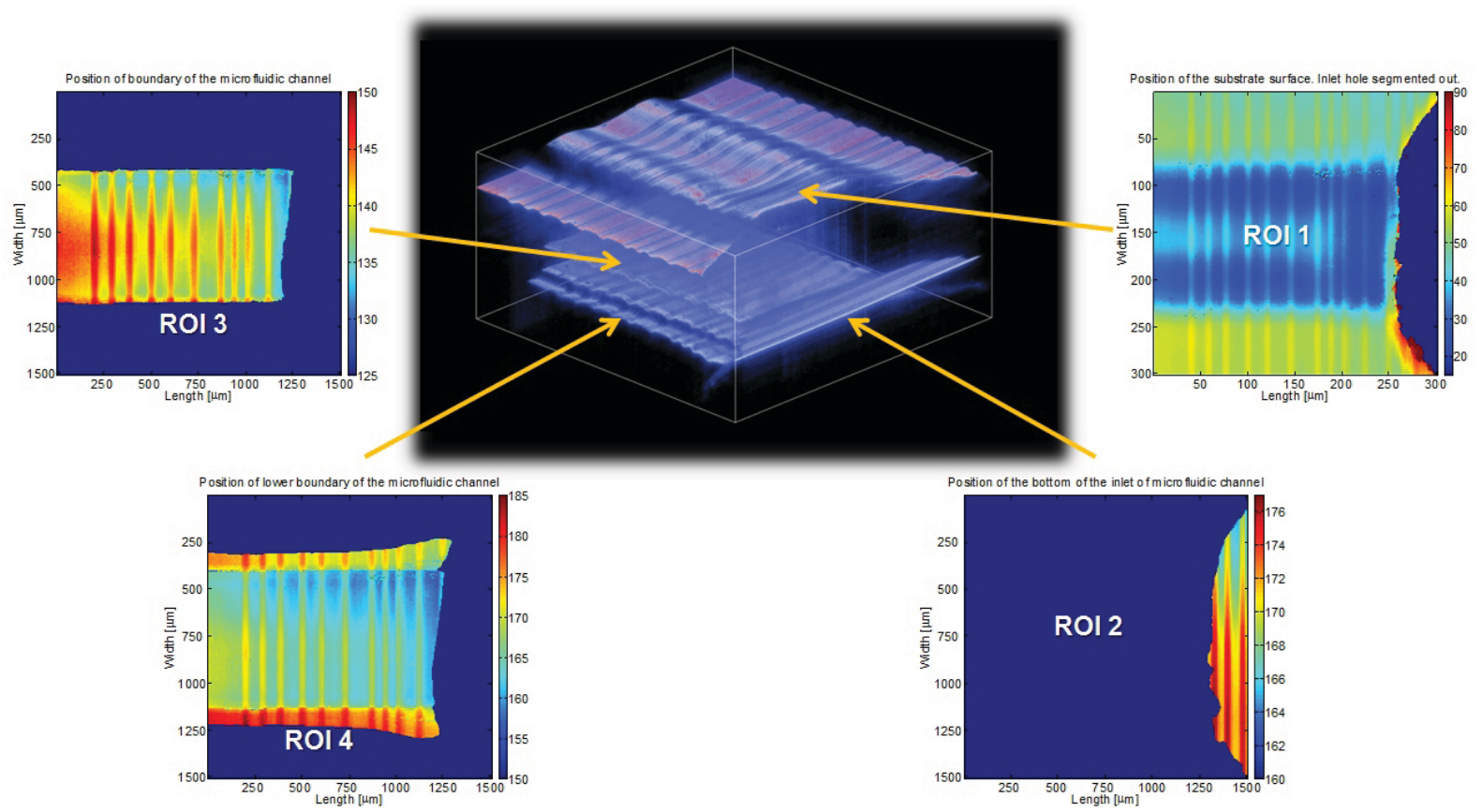

Figure 3. Volumetric reconstruction of raw measurement data (centre) and topography maps of all interfaces (around). Size of the $3 \mathrm{D}$ boundary box $1500 \times 1500 \times 272 \mu \mathrm{m}$. Values for colour maps given in microns.

Observing the raw measurement data, one can see additional artefacts. In micrometer scale measurements every factor may become an issue. Here, temperature changes in the laboratory room caused fluctuations in path length difference between interferometer's arms or possibly shrinking and stretching of polymer. Solution to this problem is presented later in the article.

\subsection{Data processing}

As described in previous section, measured data of the microfluidic chip needed some refinement to provide useful information. Main task of data processing in case of microfluidic channels is therefore transition from optical to physical structure of the sample.

The first data processing step was to acquire depth position of all sample interfaces. Each B-scan was vertically divided into separate regions of interest (see Figure 2 above). For each region maximum amplitude and its position in A-scan was calculated.

The next calculation step was detection of the inlet hole. Information about its position was needed for 
accurate volume reshaping, as discussed before. Detection was done by segmenting amplitude map of the airpolymer interface (i.e., amplitude map of sample's surface) acquired in previous processing step.

In this study active contour segmentation algorithm presented by Lankton et. al. was used. ${ }^{21}$ Depending on the complexity of the interface and signal-to-noise ratio, the algorithm was used with from 200 to 400 iteration steps. The result of segmentation can be seen in Figure 4. The same segmentation algorithm was applied to amplitude maps of other interfaces. Segmented images were used as masks to leave out areas with no structural information i.e., containing noise. Topography of each interface can be seen in Figure 3 presented before.
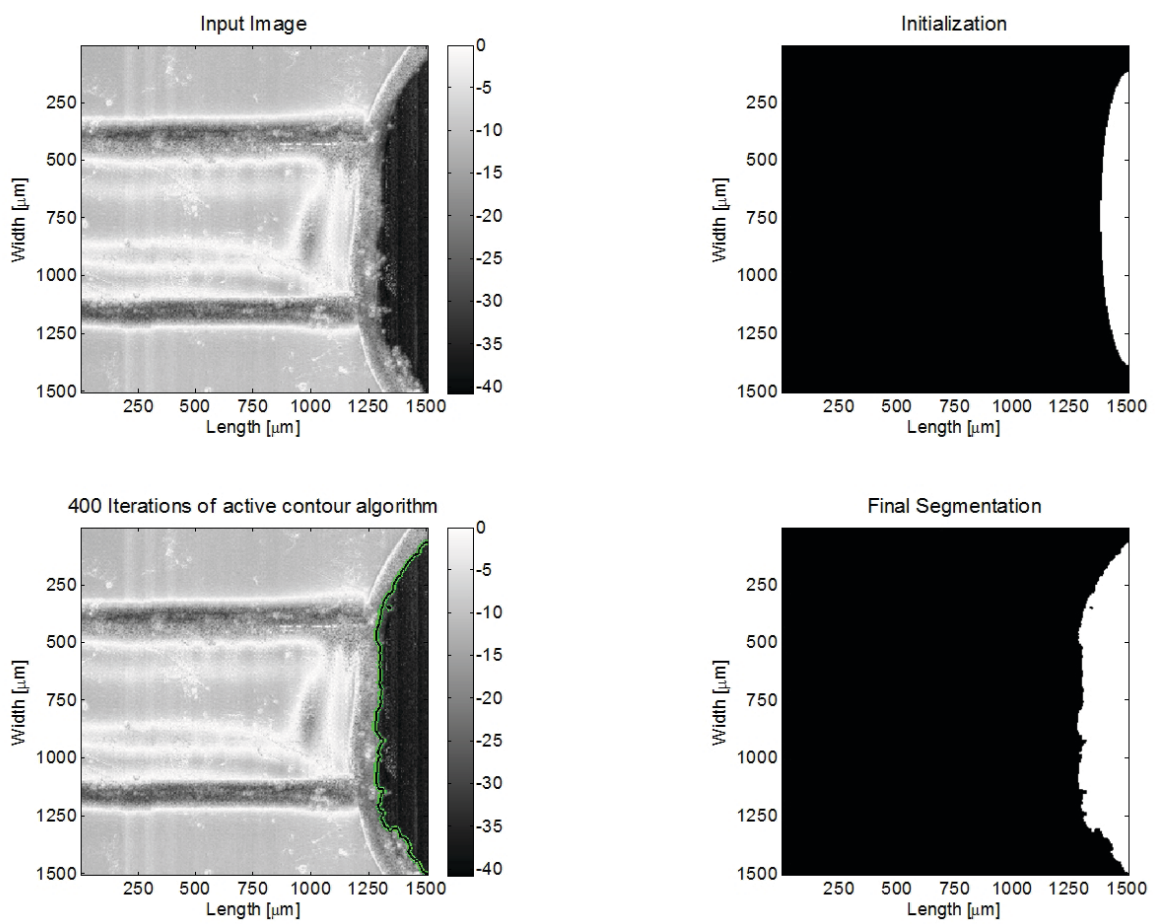

Figure 4. Channel's inlet hole detection using amplitude map of sample's surface and active contour segmentation algorithm. Colour map values in dB.

Knowing depth position of each interface, a refractive index correction could be made. Appropriate regions of each A-scan were interpolated according to their refractive index value. This is where information about inlet hole position was useful, as this region required no correction (the hole contained air). From this point, volume structure should correspond with physical structure of the sample. However, as mentioned in previous section, in case of our measurement volume still contained environmental artefacts.

Finally, the environmental artefact problem could be addressed. Once again first interface/sample surface was used. Topography map was convolved with non-uniform high/low-pass filter kernel in such manner that the high-pass side of kernel was oriented along temperature changes. Resulting map was divided by low-pass filtered initial map leaving the temperature change pattern. As surface contains channel's inlet hole, which could affect filtering, only one, borderline profile was chosen and repeated over whole area. Figure 5 shows initial surface topography map and resolved temperature caused fluctuations map.

Having temperature fluctuation map, final volume correction could be made. Correction was done by shifting each A-scan by the value obtained from the map. After all steps described above, the volume contained real physical structure of measured sample.

\section{RESULTS}

Performed measurements and data processing allowed for full, physical, volumetric reconstruction of microfluidic channel. During the data processing stage, measured data was reshaped to match the real structure of measured 

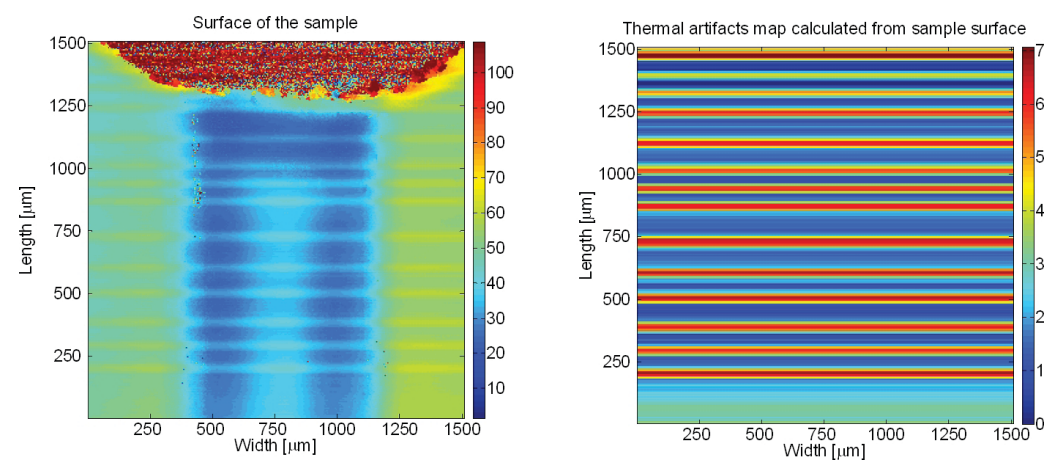

Figure 5. Surface topography map (left) and map of fluctuations caused by temperature changes (right). Values given in microns.

sample. Final volume can be seen in Figure 6. As previously, also this time, maps of interfaces existing inside sample are shown in the same figure. As expected, the bottom of the inlet hole matches the bottom of the microfluidic channel properly. Most of the temperature artefacts were also rejected.

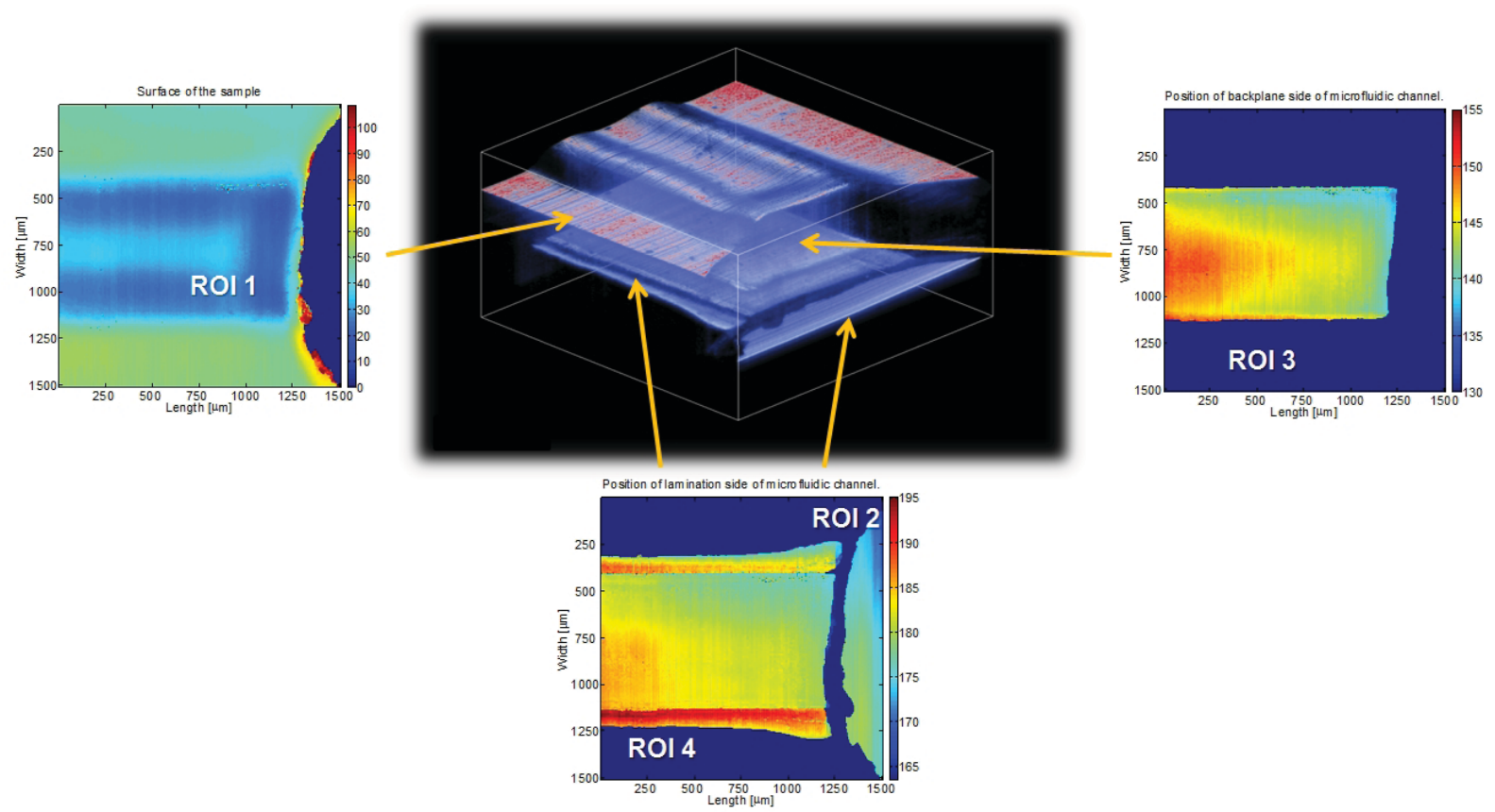

Figure 6. Result of data processing. Final volumetric reconstruction of microfluidic channel (centre) and corrected topography maps of all interfaces (around). Size of the 3D boundary box $1500 \times 1500 \times 272 \mu \mathrm{m}$. Values for colour maps given in microns.

Having complete volumetric information, one is able to derive virtually any parameter connected with structure of the channel. Standard parameters such as height, bottom and top channel width, draft angle etc. can all be calculated. In case of measured channel, average height yield $36.7 \mu \mathrm{m}$ with standard deviation of $1.4 \mu \mathrm{m}$. Widths of the top and bottom sidewalls of the measured channel are presented as plots in Figure 7 .

In addition, Figure 7 shows draft angle plot and height map of the channel. One can see that value shown by the colour map corresponds to the calculated mean value i.e, there are no significant hills and valeys. During calculation of the mean height value channel's sidewalls were not taken into account. Due to mirror reflection-like behaviour of channel's sidewalls, their position was not acquired during measurement. Instead, a straight line approximation was done using top and bottom boundaries of the channel. Draft angle i.e., the angle between 

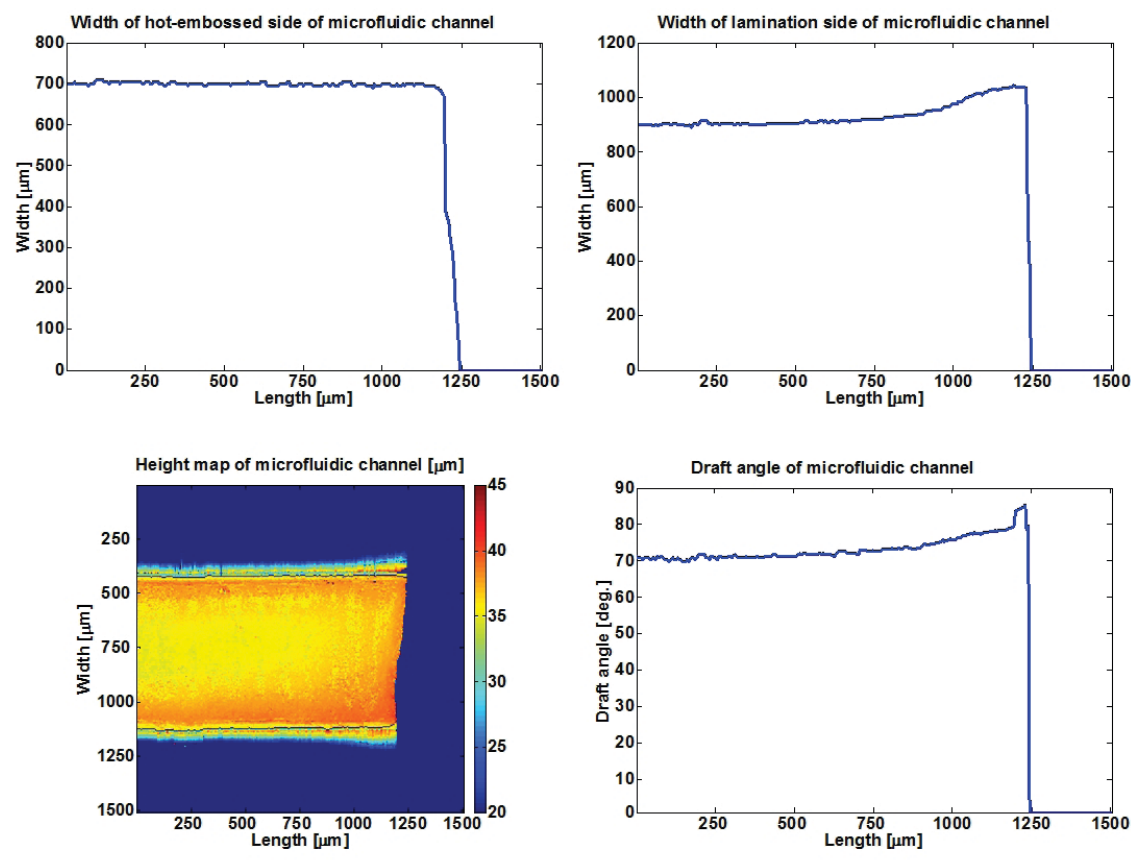

Figure 7. Widths of top and bottom sidewalls of microfluidic channel (atop) and draft angle and height map (bottom).

channel's sidewall and it's height, ${ }^{4}$ was calculated assuming trapezoidal shape of the channel. Equation 1 shows formula used in calculations.

$$
\alpha=\arctan \left[\frac{\text { bottom width }- \text { top width }}{2 * \text { channel height }}\right]
$$

The calculated mean value of the draft angle equalled 72.94 degrees. Comparing draft angle plot with channel's width plots one can say that the peak in draft angle plot (occurring at around $1200 \mu \mathrm{m}$ ) comes from non-uniform shape of the channel's inlet.

As for the lamination quality, evaluated area shows no air gaps or other features that could be described as defects and could have influence on microfluidic chip's properties and life time.

\section{CONCLUSION}

In many cases, industrial and bio-medical application come near each other. In this study, industrial application of optical coherence tomography in microfluidic channel evaluation has been presented. Despite industrial nature of presented application; aiming for evaluation of fabrication process and its result, the actual beneficiary could be life science.

Thanks to many years of constant development and research, optical coherence tomography has proven its potential to be very accurate, yet simple and cost-effective tool for quality inspection and characterization of manufactured products. For microfluidic chips evaluation OCT has many great assets. Its non-contact and non-destructive measurement nature makes it easier to avoid sample contamination. Furthermore, OCT does not require any sample preparation.

In this study we show, that OCT could be successfully utilized to fully characterize microfluidic channels. We demonstrate data processing methods and ways to compensate problems occurring during measurements. As final result, complete physical structure and spatial parameters of the channel have been presented.

As we stated in our previous publications, ${ }^{15,17}$ optical coherence tomography grown up to be mature technique. The state of the art Fourier domain OCT (FD-OCT) systems are capable of providing both high axial 
measurement resolution and very high, video-rate data acquisition speed. Spectral domain systems utilizing high speed spectrographs and offering speed up to $135 \times 10^{3} \mathrm{~A}$-scans/second have been presented. ${ }^{22}$ Swept source lasers enable $37 \times 10^{4} \mathrm{~A}$-scans/second data acquisition rates, ${ }^{23}$ while stretched pulse OCT method enabled even $5 \times 10^{6}$ A-scans/second. ${ }^{24,25}$ Introduction of supercontinuum based light sources to spectral domain OCT systems could effect with much higher axial resolution at data acquisition rates comparable to these presented so far.

In presented application, all of these properties of OCT technique, combined with roll-to-roll fabrication method, create possibility and perspective for on-line OCT measurements. Acquired data could be used in theoretical calculations and simulations (e.g., possible flow rate estimation). However, the biggest advantage comes from the fact that information obtained during such measurement may play a great role in adjustment of the fabrication process and product lifetime increase, ergo save one's time and money.

\section{ACKNOWLEDGMENTS}

The authors would like to thank Academy of Finland for financial support during the research (Grant No. 110943). Authors would like to also thank VTT Centre for Printed Intelligence in Oulu, Finland for successful cooperation and supplying samples for measurements. Finally, our thanks go to Dr Shawn Lankton for permission to use his Matlab source code for segmentation algorithm applied in this study.

\section{REFERENCES}

[1] Whitesides G. M., "The origins and the future of microfluidics", Nature, Vol 442, 368-373 (2006).

[2] Li. D. (Ed.), [Encyclopedia of Microfluidics and Nanofluidics], Springer Science+Business Media, LLC., New York, (2008).

[3] Toh A. G. G., Wang Z.F., and Ng S.H., "Fabrication of Embedded Microvalve on PMMA Microfluidic Devices through Surface Functionalization", IEEE Proc. DTIP MEMS MOEMS 2008, ISBN: 978-2-35500006-5 (2008).

[4] Chien R-D., "Hot embossing of microfluidic platform", Int. Commun. Heat Mass 33, 645-653 (2006).

[5] Ng S. H., Yeo L. P., and Wang Z. F., "Investigation on hot roller embossing process", SIMTech Tech. Rep. 10(1), 50-54 (2009).

[6] Hardt D., Ganesan B., Dirckx M., Shoji G., Thaker K., and Qi W., "Process Variability in MicroEmbossing", IMST report, Massachusetts Institute of Technology, 2005

[7] Shilpiekandula V., Burns D.J., El Rifai K., Youcef-Toumi K., Shiguang L., Reading I, Yoon S.F., "Metrology of Microfluidic Devices: A Review", Proc. 1st Int. Conf. Multi-material Micro Manufacture, 49/ICOMM2006 (2006).

[8] Huang D., Swanson E. A., Lin C. P., Schuman J. S., Stinson W. G., Chang W., Hee M. R., Flotte T., Gregory K., and Puliafito C. A., "Optical Coherence Tomography", Science 254, 1178-1181 (1991).

[9] Alarousu E., Krehut L., Prykäri T., and Myllylä R., "Study on the use of optical coherence tomography in measurements of paper properties", Meas. Sci. Technol. 16, 1131-1137 (2005).

[10] Alarousu E., "Low coherence interferometry and optical coherence tomography in paper measurements", Dr. Thesis, Faculty of Technology, University of Oulu, Oulu (2006).

[11] Prykäri T., Tuominen M., Alarousu E., Czajkowski J., and Myllylä R., "Investigating coating layer of extrusion coated paper by Optical Coherence Tomography", Proc. 2nd Int. Top. Meet. Optical Sensing and Artificial Vision - OSAV'2008, 410-417 (2008).

[12] Prykäri T., Alarousu E., Kuivaniemi J., Czajkowski J., and Myllylä R.:" Ultra-high resolution optical coherence tomography in paper characterization", Proc. 2nd Int. Top. Meet. Optical Sensing and Artificial Vision - OSAV'2008, 111-117 (2008).

[13] Czajkowski J., "Design and development of interferometer for ultra-high resolution optical coherence tomography system", M. Sc. Thesis, Faculty of Microsystem Electronics and Photonics, Wroclaw University of Technology, Wroclaw (2008). 
[14] Czajkowski J., Prykäri T., Alarousu E., Fabritius T., and Myllylä R.:"Ultra-high resolution optical coherence tomography as a method of quality inspection for printed electronics encapsulation", Northern Optics 2009 Conference: Program and book of abstracts, 113 (2009).

[15] Prykäri T., Czajkowski J., Alarousu E., and Myllylä R.: "Optical Coherence Tomography as an Accurate Inspection and Quality Evaluation Technique in Paper Industry", Opt.Rev. 17(3), 218-222 (2010).

[16] Alarousu E., Prykäri T., Palosaari J., and Myllylä R., "Ti:Sapphire Based Laser Source for Ultra-High Resolution Optical Coherence Tomography Imaging of Paper", Proc. 5th ODIMAP , 210-215 (2006).

[17] Czajkowski J., Prykäri T., Alarousu E., Palosaari J., and Myllylä R., "Optical Coherence Tomography as a Method of Quality Inspection for Printed Electronics Products", Opt. Rev. 17(3), 257-262 (2010).

[18] Stifter D., "Beyond biomedicine: a review of alternative applications and developments for optical coherence tomography", Appl. Phys. B 88, 337-357 (2007).

[19] VTT Centre for Printed Intelligence: Annual report (2009).

[20] Hosek P., Prykäri T., Alarousu E., and Myllylä R., "Application of LabVIEW: Complex Software Controlling of System for Optical Coherence Tomography", J. Assoc. Lab. Autom. 14, 59-68 (2009).

[21] Lankton S., Nain D., Yezzi A., and Tannenbaum A., "Hybrid geodesic region-based curve evolutions for image segmentation", Proc. SPIE, Vol. 6510, 65104U (2007)

[22] Grulkowski I., Gora M., Szkulmowski M., Gorczynska I., Szlag D., Marcos S., Kowalczyk A., and Wojtkowski M., "Anterior segment imaging with Spectral OCT system using a high-speed CMOS camera", Opt. Express 17(6), 4842-4858 (2009).

[23] Huber R., Adler D. C., and Fujimoto J. G., "Buffered Fourier domain mode locking: unidirectional swept laser sources for optical coherence tomography imaging at 370,000 lines/s", Opt. Lett. 31(20), 2975-2977 (2006).

[24] Park Y., Ahn T-J., Kieffer J-C., and Azaña J., "Optical frequency domain reflectometry based on real-time Fourier transformation", Opt. Express 15(8), 4597-4616 (2007).

[25] Moon S., and Kim D. Y., "Ultra-high-speed optical coherence tomography with a stretched pulse supercontinuum source", Opt. Express 14(24), 11575-11584 (2006). 